\title{
The power of imaging to understand extracellular vesicle biology in vivo
}

\author{
Frederik J. Verweij ${ }^{1,2,31 凶}$, Leonora Balaj ${ }^{3,30}$, Chantal M. Boulanger ${ }^{4,30}$, David R. F. Carter ${ }^{5,6,30}$, \\ Ewoud B. Compeer ${ }^{7,30}$, Gisela D'Angelo ${ }^{8,30}$, Samir El Andaloussi ${ }^{6,9,30}$, Jacky G. Goetz ${ }^{10,30}$, \\ Julia Christina Gross $\circledR^{11,30}$, Vincent Hyenne ${ }^{10,12,30}$, Eva-Maria Krämer-Albers ${ }^{13,30}$, Charles P. Lai ${ }^{14,30}$, \\ Xavier Loyer ${ }^{4,30}$, Alex Marki ${ }^{15,30}$, Stefan Momma ${ }^{16,30}$, Esther N. M. Nolte-'t Hoen ${ }^{17,30}$, \\ D. Michiel Pegtel ${ }^{18,30}$, Hector Peinado ${ }^{19,30}$, Graça Raposo ${ }^{8,30}$, Kirsi Rilla ${ }^{20,30}$, Hidetoshi Tahara ${ }^{21,30}$, \\ Clotilde Théry ${ }^{22,30}$, Martin E. van Royen ${ }^{23,30}$, Roosmarijn E. Vandenbroucke ${ }^{24,30}$, \\ Ann M. Wehman ${ }^{25,30}$, Kenneth Witwer ${ }^{26,30}$, Zhiwei Wu ${ }^{27,28,29,30}$, Richard Wubbolts $\mathbb{1}^{17,30}$ and \\ Guillaume van Niel (1) 1,2,31凶
}

Extracellular vesicles (EVs) are nano-sized lipid bilayer vesicles released by virtually every cell type. EVs have diverse biological activities, ranging from roles in development and homeostasis to cancer progression, which has spurred the development of EVs as disease biomarkers and drug nanovehicles. Owing to the small size of EVs, however, most studies have relied on isolation and biochemical analysis of bulk EVs separated from biofluids. Although informative, these approaches do not capture the dynamics of EV release, biodistribution, and other contributions to pathophysiology. Recent advances in live and high-resolution microscopy techniques, combined with innovative EV labeling strategies and reporter systems, provide new tools to study EVs in vivo in their physiological environment and at the single-vesicle level. Here we critically review the latest advances and challenges in EV imaging, and identify urgent, outstanding questions in our quest to unravel EV biology and therapeutic applications.

K nowledge of EV biogenesis pathways and biological activities has grown rapidly in the past decade ${ }^{1}$ (Fig. 1a,b). EVs are membrane-enclosed structures that are released into the extracellular milieu by all organisms and cell types studied so far. EVs are a diverse family in which subtypes have been defined based on subcellular origin, size, and composition: endosome-derived vesicles (including multivesicular endosome-derived exosomes with a diameter of $50-150 \mathrm{~nm}$ and secretory autophagosome-derived EVs); ectosomes and other microvesicles that bud from the plasma membrane (PM) as small as exosomes or up to several $\mu \mathrm{m}$ in size; midbody remnants released by dividing cells (Box 1); migrasomes trailing behind migrating cells ${ }^{2,3}$; apoptotic bodies dislodged from

'Université de Paris, Institute of Psychiatry and Neuroscience of Paris (IPNP), INSERM U1266, Paris, France. ${ }^{2}$ GHU Paris Psychiatrie et Neurosciences, Hôpital Sainte Anne, Paris, France. ${ }^{3}$ Department of Neurosurgery, Massachusetts General Hospital, Harvard Medical School, Boston, MA, USA. ${ }^{4}$ Université de Paris, PARCC, INSERM, Paris, France. ${ }^{5}$ Department of Biological and Medical Sciences, Faculty of Health and Life Sciences, Oxford Brookes University, Oxford, UK. ${ }^{6}$ Evox Therapeutics Limited, Oxford Science Park, Oxford, UK. ' Kennedy Institute of Rheumatology, NDORMS, University of Oxford, Oxford, UK. ${ }^{8}$ Institut Curie, PSL Research University, CNRS, UMR144 Cell Biology and Cancer, Paris, France. ${ }^{\circ}$ Clinical Research Center, Department of Laboratory Medicine, Karolinska Institutet, Stockholm, Sweden. ${ }^{10}$ INSERM UMR_S1109, Tumor Biomechanics Lab, Université de Strasbourg, Fédération de Médecine Translationnelle de Strasbourg (FMTS), Equipe Labellisée Ligue contre le Cancer, Strasbourg, France. "'Health and Medical University, Potsdam, Germany. ${ }^{12}$ CNRS SNC5055, Strasbourg, France. ${ }^{13}$ Johannes Gutenberg-Universität Mainz, Institute of Developmental Biology and Neurobiology, Mainz, Germany. ${ }^{14}$ Institute of Atomic and Molecular Sciences, Academia Sinica, Taipei, Taiwan. ${ }^{15}$ La Jolla Institute for Allergy and Immunology, La Jolla, CA, USA. ${ }^{16}$ Institute of Neurology (Edinger Institute), Goethe-University, Frankfurt am Main, Germany. ${ }^{17}$ Department of Biomolecular Health Sciences, Faculty of veterinary medicine, Utrecht University, Utrecht, the Netherlands. ${ }^{18}$ Amsterdam UMC, Vrije Universiteit Amsterdam, Pathology, Cancer Center Amsterdam, Amsterdam, the Netherlands. ${ }^{19}$ Microenvironment and Metastasis Laboratory, Molecular Oncology Programme, Spanish National Cancer Research Center (CNIO), Madrid, Spain. ${ }^{20}$ University of Eastern Finland, Institute of Biomedicine, Kuopio, Finland. ${ }^{21}$ Department of Cellular and Molecular Biology, Graduate School of Biomedical and Health Sciences, Hiroshima University, Hiroshima, Japan. ${ }^{22}$ Institut Curie, PSL Research University, INSERM U932, Immunity and Cancer, Paris, France. ${ }^{23}$ Erasmus MC, Department of Pathology, Rotterdam, the Netherlands. ${ }^{24}$ VIB Center for Inflammation Research and Department of Biomedical Molecular Biology, Ghent University, Ghent, Belgium. ${ }^{25}$ Department of Biological Sciences, University of Denver, Denver, CO, USA. ${ }^{26}$ Department of Molecular and Comparative Pathobiology and Neurology and the Richman Family Precision Medicine Center of Excellence in Alzheimer's Disease, Johns Hopkins University School of Medicine, Baltimore, MD, USA. ${ }^{27}$ Center for Public Health Research, Medical School, Nanjing University, Nanjing, China. ${ }^{28}$ State Key Laboratory of Analytical Chemistry for Life Science, Nanjing University, Nanjing, China. ${ }^{29}$ Medical School, Jiangsu Key Laboratory of Molecular Medicine, Nanjing University, Nanjing, China. ${ }^{30}$ These authors contributed equally: Leonora Balaj, Chantal M. Boulanger, David R. F. Carter, Ewoud B. Compeer, Gisela D'Angelo, Samir El Andaloussi, Jacky G. Goetz, Julia C. Gross, Vincent Hyenne, Eva-Maria Krämer-Albers, Charles Lai, Xavier Loyer, Alex Marki, Stefan Momma, Esther Nolte 't Hoen, D. Michiel Pegtel, Hector Peinado, Graça Raposo, Kirsi Rilla, Hidetoshi Tahara, Clotilde Théry, Martin E. van Royen, Roosmarijn Vandenbroucke, Ann M. Wehman, Kenneth Witwer, Zhiwei Wu, Richard Wubbolts. ${ }^{31}$ These authors jointly supervised this work: Frederik J. Verweij, Guillaume van Niel.凶e-mail: frederikverweij@gmail.com; guillaume.van-niel@inserm.fr 
a

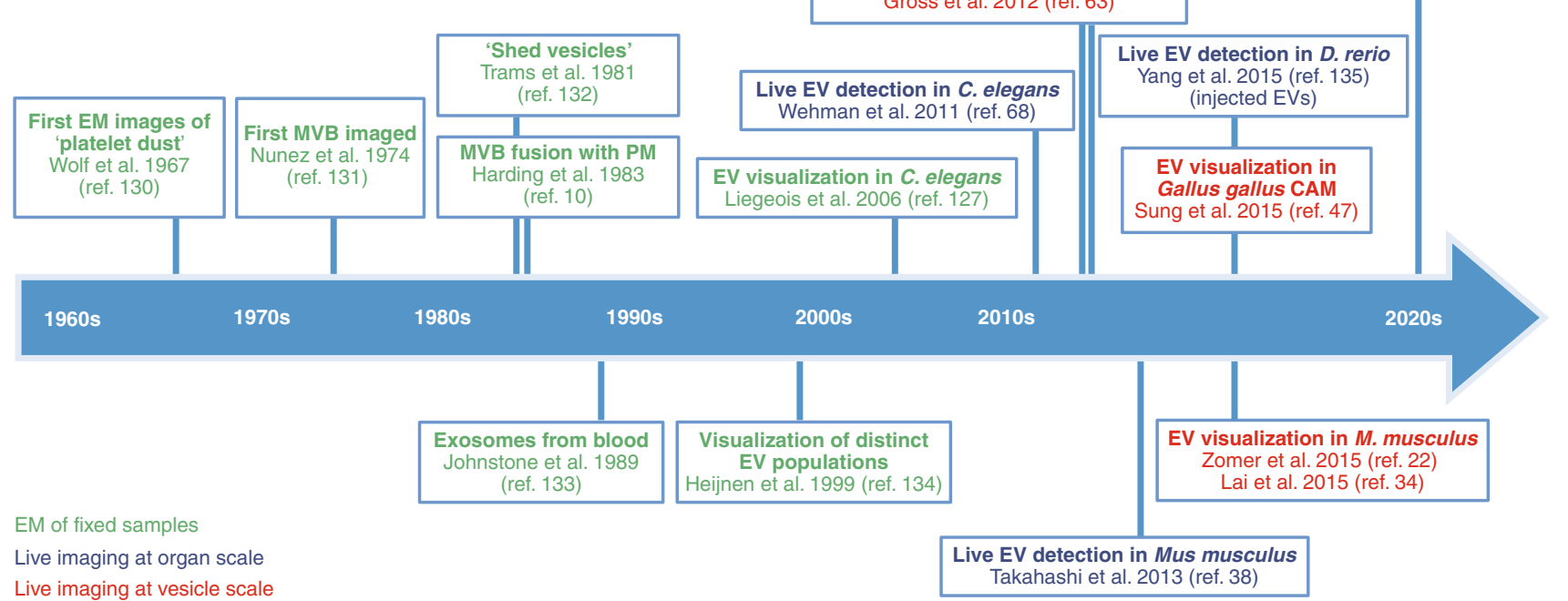

b

$10 \mathrm{~cm}$

Fig. 1 | Timeline of EV imaging milestones and broad overview of microscopy techniques to resolve EVs at different scales. a, Timeline of imaging milestones in EV research. EM, electron microscopy. Refs. ${ }^{210,17,9,22,34,38,47,63,68,128,129,131-136}$. b. Schematic of the resolution range of different microscopic approaches to resolve EVs at increasing resolution.

dying and disintegrating cells; and large oncosomes released by transformed cells with exaggerated membrane plasticity (Fig. 2a and Table 1). Recent discoveries reveal additional subclasses of microparticles and nanoparticles, such as exophers ${ }^{4,5}$, exomeres ${ }^{6}$, supramolecular attack particles ${ }^{7}$, and elongated particles ${ }^{8}$. Initial discoveries implicated EVs in cellular adherence (as 'adherons') ${ }^{9}$ and clearance ${ }^{10}$ in the early 1980s, and in immune regulation in the mid-1990s ${ }^{11}$. EVs also play crucial roles in neurodegenerative diseases, cancer progression, metabolic homeostasis, angiogenesis, inflammation, neuronal plasticity, migration, trophic support, and pathogenic infections $s^{12-15}$. These roles are primarily supported by the capacity of EVs to shuttle molecules from one cell to another.

Despite the clear importance of EV biology, EV research faces challenges imposed by the small size and heterogeneity of EVs. Most studies have used bulk separation and characterization of heterogeneous populations of EVs from biological fluids or extended, large-scale in vitro cell cultures. These approaches allow robust characterization $^{16}$ at the population level-for example, size and molecular profiles-but removing EVs from their context precludes insight into subcellular origin, release and uptake dynamics, and half-life. Separation can also disrupt fragile components such as branched glycans (Box 1), potentially altering EV functionality. Furthermore, studies in two-dimensional (2D) monocultures do not necessarily reflect what occurs in vivo.

Recent advances in live and high-resolution microscopy, combined with novel EV labeling strategies, now allow us to interrogate the composition and behavior of EVs at the single-vesicle level in living organisms ${ }^{17-20}$ (Fig. 1a). Functional transfer of EV proteins and RNA can also be assessed using novel reporters in vivo ${ }^{21,22}$ and in vitro ${ }^{23}$. These developments open new vistas in EV biology, providing the means to examine previously intractable issues such as assessing the lifespan of EVs in vivo. Here we review the 

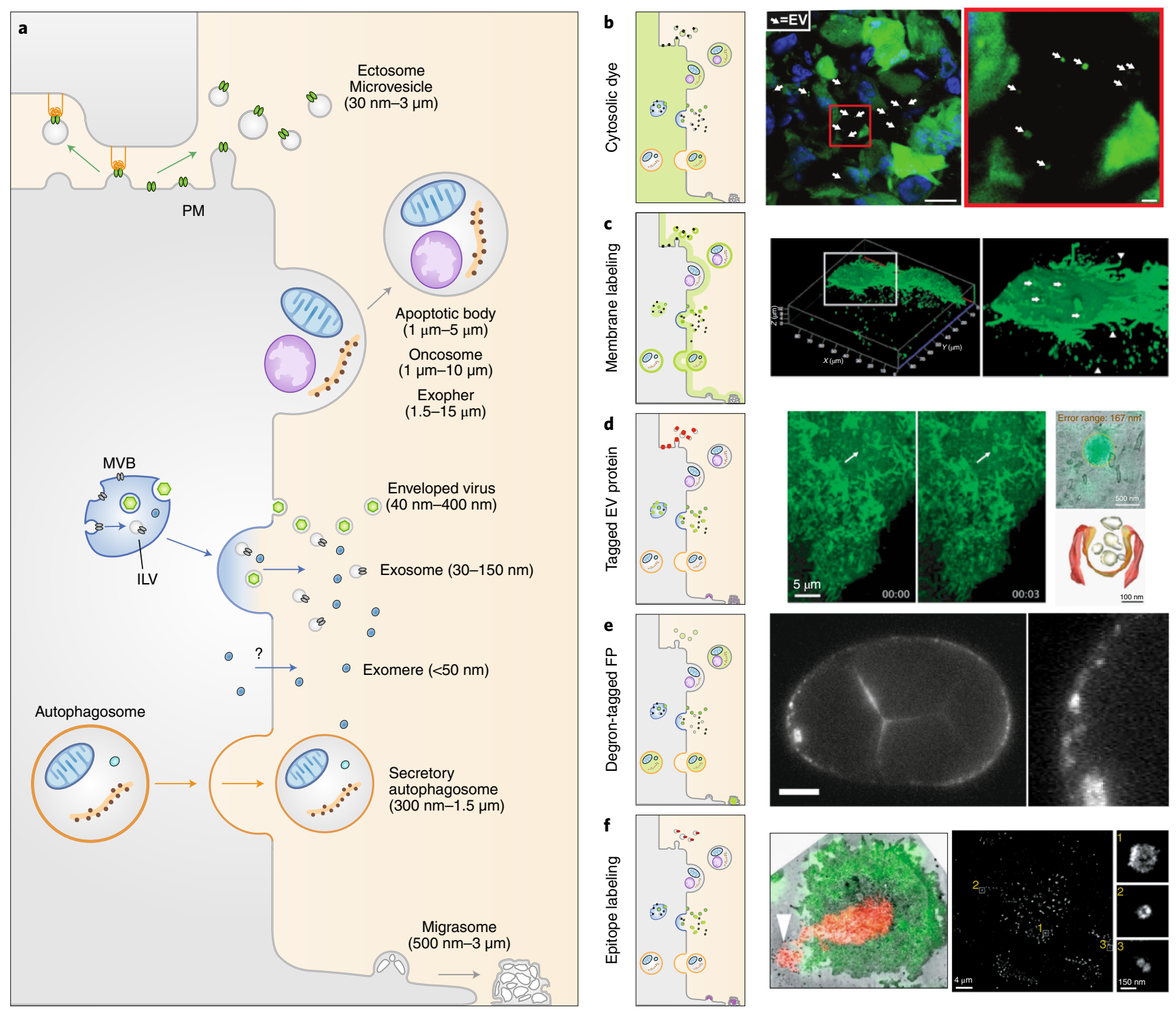

Fig. 2 | Tagging strategies to image EV production. a, EVs are diverse double-leaflet membrane-enclosed structures generated from the PM (microvesicles, apoptotic bodies, oncosomes, exophers, enveloped viruses, and migrasomes), from endosomal compartments (exosomes and enveloped retroviruses), and from autophagic compartments (secretory autophagosomes). The origin of exomeres is still uncertain. b-d, Tagging strategies to image EVs. Cytoplasmic labeling facilitates pan-EV tagging by labeling the cell cytosol and the lumen of any EVs (b). Right, large EVs released from MDA-MB-231 cells expressing Dendra2 in mice mammary glands ${ }^{22}$. Arrows, EVs. Scale bars: $10 \mathrm{~mm}$ (left image), $1 \mathrm{~mm}$ (right image). Membrane labeling tags multiple EV subtypes (c). Right, confocal microscopy of live PalmGFP-expressing $293 \mathrm{~T}$ cells releasing EVs ${ }^{34}$. Arrows, bud-like structure from the surface; arrowheads, processes extending from cells. Expressing tagged cargo proteins allows the tracking of EV subtypes (d). Right, live imaging of a burst of CD63-pHluorin fluorescence at the HeLa cell surface (arrows, fusion event), overlaid using CLEM (top right image) to observe an MVB fusing with the PM to release exosomes (bottom right image) ${ }^{49}$. e, Expression of degron-tagged fluorescent proteins allows EV tagging while cytosolic fluorescence in the source cell is degraded. Right, PH::CTPD-labeled EVs released from the unlabeled PM in C. elegans ${ }^{51}$. Scale bar, $10 \mu \mathrm{m}$. f, Targeting of EV surface proteins by antibodies. Right, optical-EM correlation of $M$. musculus $\mathrm{T}$ cell that released EVs (red) ${ }^{53}$. Arrowhead, released microvesicles. Single EV imaging by dSTORM analysis of antibody staining ${ }^{54}$ (right image and insets).

state-of-the-art in EV labeling and tracking in animal model systems. We identify pitfalls and propose solutions and best practices. Finally, we discuss how recent advances in imaging can address open questions in EV biology, from biogenesis to uptake and function, thereby enhancing the development of EV therapeutics.

\section{Tagging strategies, microscopy technology, and animal models}

Labeling strategies that allow imaging with subcellular resolution are necessary for imaging EVs. In recent years, several such strategies or applications have been developed, ranging from novel lipid dyes (Box 1) to luminal dyes and genetic labeling (Table 2 and Fig. 2).

Lipid dyes. Lipid dyes (for example, PKH67, DiR/DiD, MemGlow) have been widely applied to label EVs with various excitation and emission wavelengths ${ }^{24}$, including the infrared range for greater penetration through tissues for in vivo studies. However, the application of lipophilic dyes to study EVs is complicated by unbound dye, aggregate and micelle formation, promiscuous labeling of non-EV particles, and the long half-life ${ }^{25}$. Labeling protocols should 
Table 1 | EVs and particles

\begin{tabular}{|c|c|c|c|c|}
\hline Name & Size & $\begin{array}{l}\text { Acronyms and other } \\
\text { names }\end{array}$ & Origin & Features \\
\hline Exosomes & $30 \mathrm{~nm}-150 \mathrm{~nm}$ & $\begin{array}{l}\text { Tolerosomes, } \\
\text { prostasomes }\end{array}$ & $\begin{array}{l}\text { MVBs, late or recycling } \\
\text { endosomes, amphisomes }\end{array}$ & $\begin{array}{l}\text { Lipid bilayer; contains proteins, } \\
\text { genetic material, metabolites }\end{array}$ \\
\hline Apoptotic bodies & $1 \mu \mathrm{m}-5 \mu \mathrm{m}$ & Apoptotic blebs & PM & $\begin{array}{l}\text { Lipid bilayer; contains proteins, } \\
\text { cytosolic components, organelles, } \\
\text { nuclear fragments }\end{array}$ \\
\hline Oncosomes & $100 \mathrm{~nm}-400 \mathrm{~nm}$ & None & PM & $\begin{array}{l}\text { Lipid bilayer; contains oncoproteins, } \\
\text { genetic material, oncometabolites }\end{array}$ \\
\hline Exomeres & $<50 \mathrm{~nm}$ & None & ND & $\begin{array}{l}\text { Might lack a lipid bilayer; contains } \\
\text { proteins such as argonaute and } \\
\text { APP, lipids, and nucleic acids }\end{array}$ \\
\hline Exophers & $1.5-15 \mu \mathrm{m}$ & None & PM & $\begin{array}{l}\text { Lipid bilayer; contains metabolic } \\
\text { waste, protein aggregates, } \\
\text { organelles }\end{array}$ \\
\hline Secretory autophagosomes & $300 \mathrm{~nm}-1.5 \mu \mathrm{m}$ & Mitovesicles? & Autophagic pathway & $\begin{array}{l}\text { Lipid bilayer; contains } \\
\text { cytoplasmic contents, excess or } \\
\text { damaged proteins, organelles, } \\
\text { microorganisms }\end{array}$ \\
\hline
\end{tabular}

EVs comprise a heterogeneous population of membrane vesicles. Their sizes vary between $<50 \mathrm{~nm}$ and $>5 \mu \mathrm{m}$. They can originate from the PM, or the endosomal or autophagic pathways. ND, not determined.

therefore limit dye concentrations during labeling, remove free dye after labeling, include appropriate controls (for example, 'dye only' control in EV solvent), and consider using multiple differentially stained EV populations to demonstrate absence of dye transfer or vesicle aggregation after co-isolation ${ }^{26}$. Recently, MemGlow ${ }^{27}$ was reported to be brighter and less prone to aggregate formation compared with traditional lipid dyes ${ }^{19}$.

Lipid dyes can be applied directly to producer cells followed by EV isolation ${ }^{19}$. However, it is unknown whether cell labeling affects EV release or function, or equally labels EV subtypes. Lipid dyes might also affect membrane-membrane fusion, fluidity of membrane proteins, membrane stiffness and EV size ${ }^{28}$. As the half-life of lipid dyes greatly exceeds that of $\mathrm{EVs}^{29,30}$, EV degradation after cellular uptake can be masked by recycling and redistribution of fluorescent dye. Therefore, lipophilic dye labeling of EVs may be best suited for short-term studies ${ }^{31}$.

Dye labeling of the EV lumen. Dyes such as carboxyfluorescein diacetate succinimidyl ester (CFDA-SE) and calcein-AM label proteins in the EV lumen ${ }^{30,32}$. Their dependence on luminal esterases for conversion into a fluorescent product may produce fewer false-positive EV signals than lipophilic dyes but probably restricts labeling to a subpopulation of esterase-containing $\mathrm{EVs}^{33}$.

Fluorescent and bioluminescent protein EV reporters. Various genetically encoded reporters have been developed to label all EVs or subtypes (Box 1) using fluorescence or bioluminescence. Labeled proteins expressed in the cytosol can be shuttled into the lumen of both exosomes and ectosomes (Fig. 2b) ${ }^{22}$. Addition of a palmitoylation signal associates the reporter with the inner leaflet (Box 1) of PM-derived EVs in vivo (Fig. 2c) ${ }^{34}$. For labeling of specific EV subtypes, reporters (including GFPs, RFPs, and the bioluminescent ThermoLuc) can be attached to EV cargos (for example, syntenin or tetraspanin (TSPAN; Box 1) family members (TSPAN4, CD63, CD81 and CD9) $)^{2,17,19,26,35}$, of which CD63 is most widely used). Alternative scaffolds and double labeling strategies ${ }^{36}$ can be considered to permit subtype detection. In contrast to fluorescent proteins, bioluminescent proteins emit signal after substrate addition with a high signal-to-noise ratio but comparatively lower spatiotemporal resolution ${ }^{37}$. Therefore, bioluminescence-based reporters (gLuc-lactadherin, GlucB) are predominantly used in small animal models to track EV biodistribution at whole-animal and organ 


\section{Box 1 | Glossary}

Glycan extended trees. Protein modification involving attached polymerized glycans that possess structural and/or modulatory function (for example, ligand binding).

Lipid membrane dye. Lipophilic fluorescent dye that integrates in lipid membranes.

EV subtype. EV with specific subcellular origin, size, and/or composition (Table 1).

Tetraspanin. Family of membrane proteins with four transmembrane domains enriched in EVs.

Inner leaflet. Cytosol- or EV-lumen-facing layer of a lipid bilayer.

Fluorescent complementation. A technology used to validate protein interactions through the association of complementary fluorescent protein fragments attached to components of the same macromolecular complex.

Steric hindrance. Here, spatial extent of an exogenous label preventing native interaction(s) of the labeled protein.

EV cargo. Any molecule (lipid, protein, metabolite, genetic material) shuttled within or on EVs.

CLEM. Imaging technique to correlate (live) light microscopy with ultrastructural information obtained on the same sample after fixation.
Diffraction limit. Theoretical limit of optical microscopes to distinguish objects separated by a lateral distance less than half of the wavelength used.

Photobleaching. Photon-induced alteration of a fluorophore that causes it to permanently lose its ability to fluoresce.

Phototoxicity. Photon-induced damage to cellular macromolecules that impairs sample physiology.

Intraluminal vesicles. Vesicles formed inside endosomes and precursors of canonical exosomes (Table 1).

3D microenvironment. Local environment surrounding a cell, consisting of ECM, soluble factors, and other cells.

Gene traps. Here, insertion of fluorescent tag such that the labelled protein is expressed under its endogenous promoter.

Lectins. Saccharide binding proteins.

Midbody remnants. Condensed membrane structure derived from the intercellular bridge that is left over after cell division.

V-ATPase. Transmembrane proton pump functioning to acidify intracellular compartments.

Back-fusion. Process in which ILVs or internalized EVs fuse with the late-endosomal limiting membrane, exposing their lumen to the cytosol and delivering their luminal content to the cytoplasm of recipient cells. scales $^{38,39}$ (Table 2). More recently, a third category of EV reporter using bioluminescence resonance energy transfer (BRET) has been described (PalmGRET), allowing EV biodistribution analysis and in vivo quantification from whole animal to super-resolution without requiring multiple reporters ${ }^{40}$.

Excitingly, genetic labeling allows access to the entire fluorescent protein toolbox, including photoswitching and photoactivation, biosensors and bimolecular fluorescent complementation (Box 1). However, genetic labeling also comes with challenges. Labeling transmembrane proteins might disrupt conformation or cause steric hindrance (Box 1) of ligand-receptor interaction and organotropism $^{41-43}$. EV surface-associated reporters may also be prone to proteolytic cleavage ${ }^{44}$, removing the signal ${ }^{45}$. Reporter overexpression may affect cellular signaling, EV cargo loading, or endogenous EV production and trafficking. Although a recent study demonstrated that CD63-GFP labeling of EVs only minimally perturbed the EV proteome ${ }^{26}$, other studies reported alterations in endolysosomal trafficking ${ }^{46}$, suggesting context-specific effects. Overexpression may also misdirect the reporter protein to unintended EV subtypes. Moreover, the amount of fluorescence emitted by the producing cell will ordinarily overpower the fluorescent signal of small EVs (approximately one millionth of the cell volume) in the immediate vicinity. One solution is the use of $\mathrm{pH}$-sensitive fluorophores (for example, pHluorin), which are quenched in acidic cellular organelles but detected upon EV release, as successfully applied in vitro ${ }^{47-50}$ and in vivo ${ }^{17}$ (Fig. 2d). A second strategy is degron tagging, whereby cytosolic signal in the producing cell is degraded, while the signal in EVs persists ${ }^{51}$ (Fig. 2e).

Epitope targeting of EV surface proteins. EV-enriched surface proteins and glycans can be targeted to visualize and characterize EVs in live and fixed cells (Fig. 2f). Pre-labeling of glycans on the PM with fluorescent hyaluronic acid binding complex (fHABC) allows live visualization of EV budding and fission from the cell surface ${ }^{52}$. Fluorescently labelled antibody fragments, such as nanobodies or fragment antigen-binding (Fab) domains, can also target EV-enriched proteins, with the advantages of eliminating the need for a secondary antibody and their smaller size compared to intact immunoglobulins. These strategies are compatible with most microscopy approaches ${ }^{53}$ and allow imaging at single-EV resolution ${ }^{54}$. With these tags, imaging EVs near the producing cells can be difficult if the epitope is present on both EVs and the PM. Depending on the resolving power of the imaging modality, the use of EV capture ${ }^{55}$ or immobilization strategies ${ }^{56}$ may be necessary.

A 'one size fits all' EV reporter does not exist (yet), and a particular reporter should be chosen based on the biological question and available imaging equipment. The specificity of the strategies to label EVs should preferably be validated with super-resolution and/ or ultrastructural techniques. Along these lines, several recent studies have used combinations of correlative light and electron microscopy (CLEM; Box 1), immuno-electron microscopy (IEM), and/or scanning electron microscopy (SEM) to validate in vitro and in vivo approaches $^{17,19,48,49}$ (Table 2).

Microscopy. Apart from successful labeling, live imaging of EVs in vivo also requires a dedicated imaging set-up. Ideally, the set-up is suitable for deep tissue imaging while being resolutive and sensitive enough to observe EVs without inducing phototoxicity (Fig. 1b). This means relying on fast but often diffraction-limited systems (Box 1). Super-resolution microscopy (SRM) - for example, stochastic optical reconstruction microscopy (STORM) and photoactivated localization microscopy (PALM)-improve resolution to the nanometer scale, but often require fixation and are time-consuming. Other SRM approaches better suited for live-cell imaging of EV uptake and processing are structured illumination microscopy (SIM) and stimulated emission depletion microscopy (STED). All SRM techniques depend on high photon intensities, complicating detection of smaller EVs and increasing the risk of photobleaching and phototoxicity (Box 1), especially when imaging larger volumes in vivo over time. This renders some of the current SRM techniques incompatible with robust live imaging of EVs in vivo. 
Table 2 | Tagging strategies for EVs

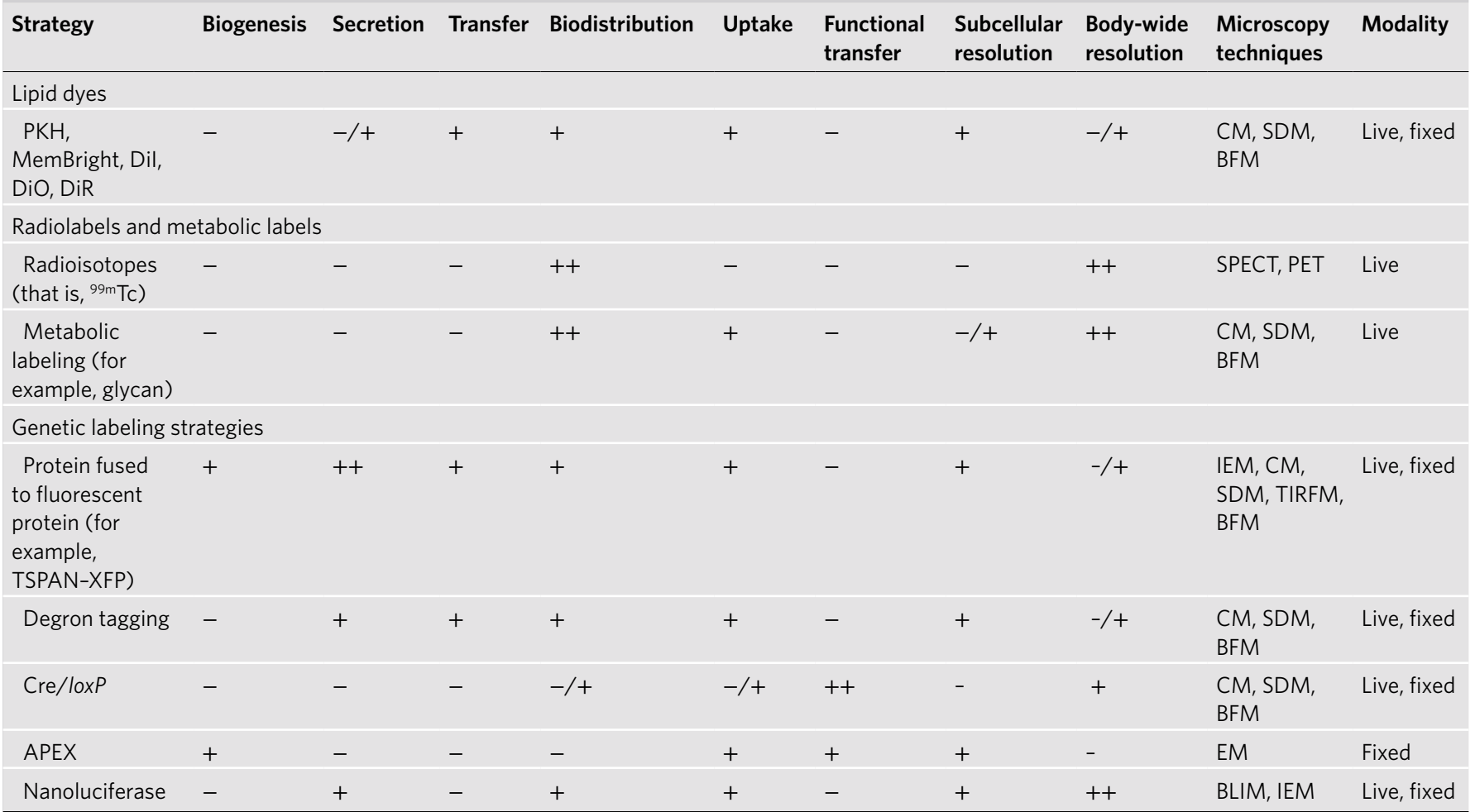

Different labeling strategies are suitable for visualizing EV (subtype) biogenesis, secretion, transfer, biodistribution, uptake, and functional (cargo) transfer, as well as for live or fixed imaging at subcellular or body-wide resolution. -, unsuitable; -/+, low suitability; +, suitable; ++, highly suitable. BFM, bright-field microscopy; BLIM, bioluminescence imaging microscopy; CM, confocal microscopy; IEM, immuno-electron microscopy; PET, positron emission tomography; SDM, spinning disk microscopy; SPECT, single-photon emission computed tomography; TIRFM, total internal reflection fluorescence microscopy.

What is the best fluorescence microscopy system to study EV biology in vivo? The answer depends on the specific research question and the physiological and pathological context (Table 3). Confocal laser scanning microscopy (CLSM) can detect EVs in the sub-200-nm range, track their uptake by living cells, and their dynamic intracellular distribution on a time scale of seconds. However, EVs will appear as a small set of pixels by light microscopy and insufficient structural detail is attained to determine EV diameter and distinguish single EVs from EV clusters, dye aggregates, or dye-labeled protein aggregates and other particles. In addition, tracking of rapidly moving EVs (for example, in circula$\operatorname{tion}^{17,19}$ ) and/or longer time-lapses require high-speed imaging with systems such as spinning disk microscopy and selective plane illumination microscopy (SPIM). These set-ups allow fast acquisition of EV movement, image larger volumes in vivo, and limit photobleaching and phototoxicity ${ }^{57}$. However, cells might be negatively affected by illumination even before they start to display morphological changes such as membrane blebbing ${ }^{57,58}$. Subtle impacts of prolonged imaging (for example, on cellular metabolic state) must be kept in mind, as they may affect EV release quantitatively and/ or qualitatively. Emerging techniques, including lattice light-sheet microscopy (LLSM), could prove instrumental to enable sustained high-resolution live imaging with minimal photobleaching and phototoxicity ${ }^{59}$.

IEM and CLEM allow validation of EV-labeling approaches; for example, to confirm proper association with intraluminal vesicles (ILVs; Box 1) $)^{17,19,48,49}$. These approaches can be used in in vitro cultures and in vivo models to study aspects of the EV lifespan like extracellular fate post-secretion or subcellular distribution in receiving cells ${ }^{17,19,60}$. Importantly, EM provides ultrastructural resolution and label-free visualization of EVs in their native environment. In addition, immunolabeling detects proteins at the single-EV or single-ILV level. However, IEM with CLEM is restricted to a posteriori imaging of fixed samples.

Model organisms. Molecular processes involved in EV biogenesis, secretion, and uptake can be studied as isolated processes using in vitro approaches. However, the physiological quantities, content, release dynamics, natural targets, and stability of EVs are likely to be affected by the 3D microenvironment (Box 1). Particularly when studying EVs in the context of intercellular communication, one of the main paradigms in the field, a relevant context is essential. The use of primary cell sources and 3D models is therefore arguably a much-needed step to provide more physiological relevance compared to 2D monocultures of immortalized cell lines in vitro.

Drosophila melanogaster is an attractive model system for studying EVs in tissue organization, development, and systemic crosstalk $^{61,62}$. Wnt and Hh-containing EVs have been observed ex vivo in D. melanogaster wing imaginal discs ${ }^{63-65}$. In addition, D. melanogaster has been used to study EV biology during mating behavior and in adaptive immunity ${ }^{66}$. Recently, an EV subpopulation from Rab11-positive multivesicular bodies (MVBs) was shown to be evolutionarily conserved in flies and human cells ${ }^{46}$. The worm Caenorhabditis elegans is also an interesting model organism to study inter-animal EV communication with fluorescently labeled $\mathrm{EVs}^{67}$ and EV biogenesis mechanisms using the ultrastructural resolution of $\mathrm{EM}^{68,69}$.

Imaging of more complex tissues, like those from vertebrates, comes with additional restraints (Table 4 and Fig. 3). The smaller the observed particle, the more important optical accessibility of the surrounding tissue becomes to reduce noise. For instance, a chorioallantoic membrane (CAM) model system allows the visualization of CD63-positive and CD44-positive EVs in vivo ${ }^{48,70}$. The zebrafish (Danio rerio), as a transparent vertebrate model, allows 
Table 3 | Microscopy methods

\begin{tabular}{|c|c|c|c|c|c|c|c|}
\hline Modalities & Resolution ( $X Y$ ) & Resolution (Z) & Illumination & Probes & $\begin{array}{l}\text { Acquisition } \\
\text { time }\end{array}$ & $\begin{array}{l}\text { Post-acquisition } \\
\text { processing }\end{array}$ & Live or fixed \\
\hline $\begin{array}{l}\text { Standard } \\
\text { fluorescence } \\
\text { microscopy }\end{array}$ & $250 \mathrm{~nm}$ & $500 \mathrm{~nm}$ & $\begin{array}{l}\text { Epi, confocal, } \\
\text { TIRF }\end{array}$ & $\begin{array}{l}\text { Conventional } \\
\text { fluorescent probes }\end{array}$ & Seconds & & Live, fixed \\
\hline SIM, airyscan & $80 \mathrm{~nm}-150 \mathrm{~nm}$ & $250 \mathrm{~nm}-350 \mathrm{~nm}$ & $\begin{array}{l}\text { Widefield (epi } \\
\text { and TIRF) }\end{array}$ & $\begin{array}{l}\text { Conventional } \\
\text { fluorescent probes }\end{array}$ & Seconds & Yes, FTT & Live, fixed \\
\hline STED & $30 \mathrm{~nm}-80 \mathrm{~nm}$ & $150 \mathrm{~nm}$ & Laser scanning & $\begin{array}{l}\text { Limited selection } \\
\text { of probes (match } \\
\text { depletion laser) }\end{array}$ & Seconds & No & $\begin{array}{l}\text { Live, fixed; } \\
\text { optimal for } \\
\text { fixed }\end{array}$ \\
\hline $\begin{array}{l}\text { Cryo-soft X-ray } \\
\text { tomography }\end{array}$ & $25 n m-40 n m$ & $30 \mathrm{~nm}$ & Widefield & none & & No & $\begin{array}{l}\text { Fixed } \\
\text { (near-native } \\
\text { state } \\
\text { vitrification) }\end{array}$ \\
\hline PALM & $20 \mathrm{~nm}$ & $50 \mathrm{~nm}$ & $\begin{array}{l}\text { Widefield (epi } \\
\text { and TIRF) }\end{array}$ & $\begin{array}{l}\text { Photoactivatible } \\
\text { fluorescent proteins }\end{array}$ & Minutes & Yes (PSF mapping) & Live, fixed \\
\hline STORM & $20 \mathrm{~nm}$ & $50 \mathrm{~nm}$ & $\begin{array}{l}\text { Widefield (epi } \\
\text { and TIRF) }\end{array}$ & Photoswitchable dyes & Minutes & Yes (PSF mapping) & Live, fixed \\
\hline LLSM & $100 \mathrm{~nm}-200 \mathrm{~nm}$ & $400 \mathrm{~nm}$ & $\begin{array}{l}\text { Multi-Bessel } \\
\text { beam plane } \\
\text { illumination }\end{array}$ & $\begin{array}{l}\text { Conventional } \\
\text { fluorescent probes }\end{array}$ & $\begin{array}{l}\text { Seconds, } \\
\text { minutes or } \\
\text { hours }\end{array}$ & $\begin{array}{l}\text { Not necessary, } \\
\text { but often tracking } \\
\text { dynamic processes }\end{array}$ & $\begin{array}{l}\text { Live, fixed; } \\
\text { optimal for live }\end{array}$ \\
\hline TEM & $<1 \mathrm{~nm}$ & $70 \mathrm{~nm}^{\mathrm{a}}$ & Electron beam & $\begin{array}{l}\text { Contrast reagent, } \\
\text { immunochemistry }\end{array}$ & Seconds & Yes & Fixed \\
\hline CLEM & $<1 \mathrm{~nm} / 150 \mathrm{~nm}^{\mathrm{b}}$ & $5 \mathrm{~nm}^{\mathrm{c}}$ & $\begin{array}{l}\text { Electron beam, } \\
\text { widefield }\end{array}$ & $\begin{array}{l}\text { Contrast reagent, } \\
\text { nanodots, and } \\
\text { fluorescent proteins }\end{array}$ & Minutes & Yes (aligning) & $\begin{array}{l}\text { Live and/or } \\
\text { fixed }\end{array}$ \\
\hline
\end{tabular}

Characteristics of imaging methods used to visualize EVs. epi, epifluorescence; FTT, fast Fourier transform; PSF, point spread function; TEM, transmission electron microscopy. ${ }^{2}$ Resolution corresponding to the thickness of the section. ${ }^{b}$ Resolution gap between electron microscopy and light microscopy data, respectively. ${ }^{C}$ Tomography from double-tilted $250 \mathrm{~nm}$ sections.

\section{Table 4 | Model systems for EV imaging}

\begin{tabular}{|c|c|c|c|c|c|c|c|c|c|c|}
\hline Model system & Biogenesis & Secretion & Transfer & Biodistribution & Uptake & $\begin{array}{l}\text { Functional } \\
\text { cargo } \\
\text { transfer }\end{array}$ & $\begin{array}{l}\text { Subcellular } \\
\text { resolution }\end{array}$ & $\begin{array}{l}\text { Relevance } \\
\text { to human } \\
\text { physiology }\end{array}$ & Cost & Throughput \\
\hline In vitro (2D) & ++ & ++ & - & - & + & + & ++ & $-/+$ & Low & High \\
\hline G. gallus $\mathrm{CAM}^{47,70}$ & + & + & + & $-1+$ & + & ++ & ++ & + & Low & Medium or low \\
\hline D. rerio ${ }^{2,7,19}$ & + & + & ++ & ++ & ++ & ++ & ++ & + & Medium & Medium \\
\hline $\begin{array}{l}\text { M. musculus, Rattus } \\
\text { norvegicus }{ }^{21,22,34,118}\end{array}$ & - & $-/+$ & + & ++ & + & ++ & $-/+$ & ++ & High & Low \\
\hline
\end{tabular}

The suitability and relevance of different model systems for EV imaging to visualize disparate aspects of EV biology at different scales. -, unsuitable; $-/+$, low suitability; +, suitable; ++, highly suitable.

continuous live imaging of the blood flow of endogenous EVs and EVs exogenously administered throughout the embryo ${ }^{17,19}$. This model has permitted the exploration of EV biology in unprecedented detail ${ }^{71}$ (Fig. 3c,d), revealing correlates of EV characteristics and function ${ }^{43}$. In mice, functional EV cargo transfer from immune to neuronal cells and between tumor cells has been observed ${ }^{21,22}$, as well as stroma-glioblastoma interactions, including microRNA (miRNA) transfer ${ }^{18,72}$. Still, live imaging of EVs in mice is currently restricted to tissues immediately adjacent to the imaging window or to larger EVs, as small EVs probably escape detection in these models ${ }^{18,22,73}$ (Fig. 3b). Imaging less accessible areas or across organs often requires organ extraction and ex vivo (post-fixation) analysis $^{74}$, and is possible only with sufficient EV accumulation over time. Moreover, sites of accumulation might not equate with sites of function. These considerations have complicated efforts to understand EV physiology in mammalian models.

Thus, each model organism has its own strengths and weaknesses. Live imaging of single EVs in D. rerio, CAM, D. melanogaster, and C. elegans is highly realistic (Fig. 3e,f) in contrast with murine models. Although mice models display a higher degree of relevance to human physiology, the applicability of non-mammalian model systems to study human pathologies remains considerable: $82 \%$ of 
a Imaging EV distribution in vivo

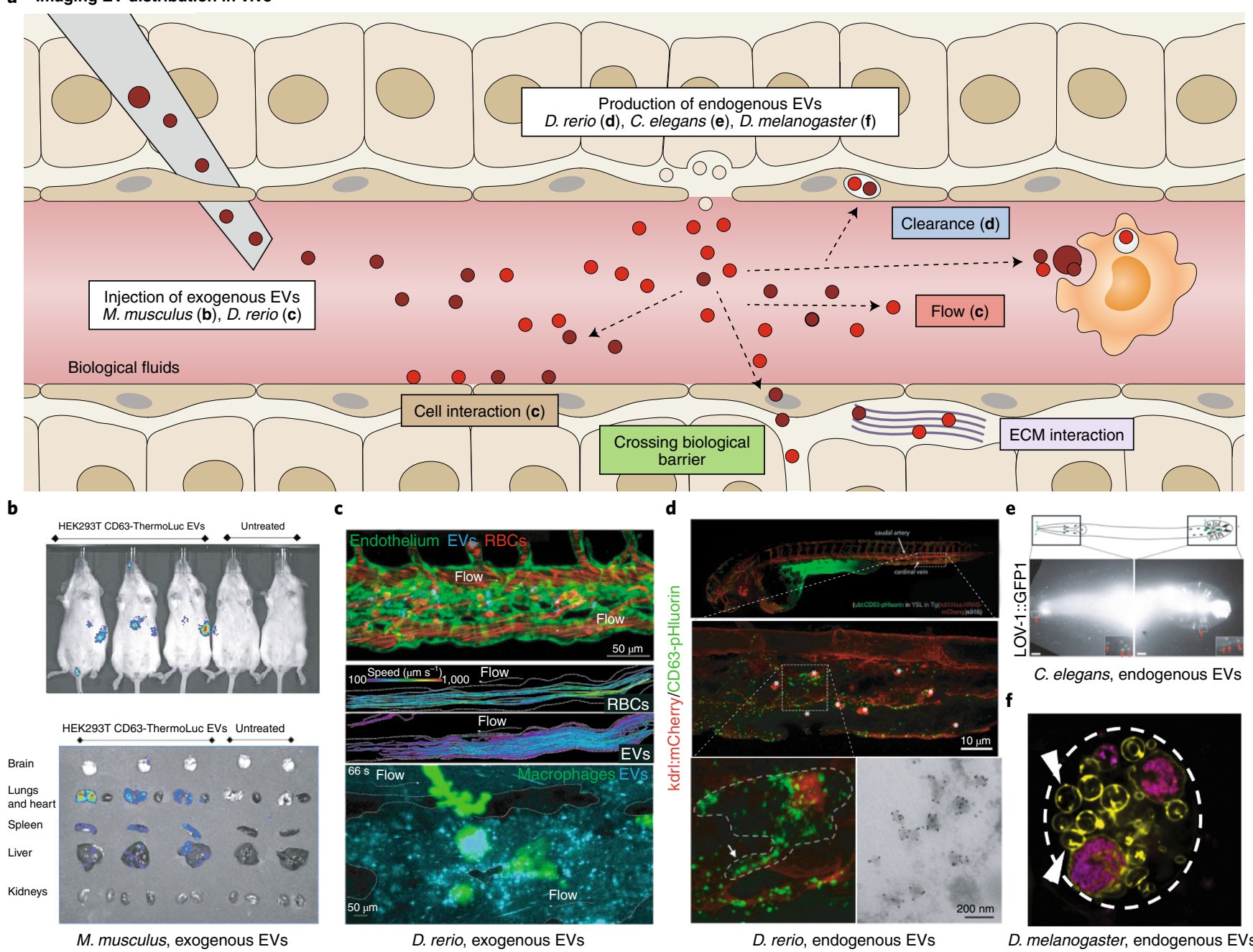

Fig. 3 | Imaging EV propagation in vivo. a, EV biodistribution can be mapped in the complex architecture of an organism after injection of labeled exogenous EVs or tagging endogenous EVs in situ. The in vivo fates of EVs (white boxes) are shown. b-d, Imaging using injected or endogenous EVs in live animals. b. EV accumulation tracked at the organ scale using CD63-ThermoLuciferase in mice ${ }^{73}$. c, EVs interacting with endothelial cells (green, top) or macrophages (green, bottom) tracked live in transparent zebrafish D. rerio; EV circulation in comparison to red blood cells (RBCs, red, middle) ${ }^{19}$. d, Endogenous EV clearance by scavenger endothelial cells and macrophages in D. rerio (top panels); inset of macrophage internalizing EVs captured from blood flow by SEC (bottom left); and IEM confirms the vesicular nature of the CD63-pHluorin signal in situ (bottom right) ${ }^{17}$. Arrow, macrophage protrusion; asterisks, macrophages. e, Fluorescently tagged EV cargo proteins track released EVs in C. elegans ${ }^{67}$. Red arrows, EVs surrounding the head and tail. Scale bars, $10 \mu \mathrm{m}$. f, Fluorescently tagged EV cargo proteins track EVs released from giant secretory MVB-like compartments in $D$. melanogaster ${ }^{130}$.

all disease-related genes are conserved in D. rerio, $75 \%$ in $D$. melanogaster, and $>65 \%$ in C. elegans ${ }^{75-77}$. For example, disease-related models of neurodegenerative pathologies and tumor development have been introduced over the past decade ${ }^{77,78}$. Therefore, while considerations regarding relevance to human pathophysiology are important for any model system, these considerations should not block the access to a superior level of insight in smaller model organisms or preclude important questions from being addressed. D. melanogaster, C. elegans and D. rerio allow fundamental investigations in cell biology and development and are often vastly superior to murine models with regard to optical accessibility, genetic amenability, costs, and suitability for medium- or high-throughput approaches (Table 4). For example, exogenous tagging of proteins and tissue-specific expression using gene traps (Box 1) is well established using the GAL4-UAS system ${ }^{79}$. Various CRISPR-Cas9 and CRISPR-Cas12a systems are available for functional studies in vivo ${ }^{80,81}$, allowing loss- and gain-of-function studies and endogenous tagging and live imaging of proteins at endogenous expression levels (although these levels may not be sufficient to reliably follow small-sized particles such as EVs). Additionally, these models can be used as 'pre-mouse' models, where mice are subsequently deployed for key validation steps. Such strategies are consistent with the ' $3 \mathrm{R}$ ' principles in animal research. The choice of model system should therefore depend on the research question, the necessary level of resolution (single versus bulk EVs), and the required throughput (Table 4).

\section{Imaging EV biogenesis, release, and distribution}

In vitro studies revealed that most cells release EVs continuously and/or adapt release in response to triggers ${ }^{49,82,83}$. Similarly, most cells can take up EVs. Bulk EV isolation from culture media thereby neglects the subset of EVs that has been released and recaptured or does not spread beyond cell-cell interfaces. Moreover, culture media components and $2 \mathrm{D}$ versus $3 \mathrm{D}$ culture methods significantly affect EV release and EV composition ${ }^{84-89}$. Furthermore, little is known about bulk or subtype EV release dynamics or its dependence on 
characteristics of specific tissues and conditions (growth, homeostasis, pathology, specific triggers). Live imaging techniques now let us grasp these temporal, spatial, and conditional EV dynamics.

Imaging EV biogenesis and release. EV s have two main subcellular origins: intracellular compartments and the PM. Although biogenesis at the PM is synonymous with release, EV release from intracellular compartments requires multiple steps, from ILV or autophagic vacuole biogenesis to organelle fusion with the cell surface (Fig. 2a).

Recent developments have enabled live visualization of PM-generated EVs by various approaches. Direct budding and fission of EVs into the extracellular milieu has been visualized in living cells after PM labeling with fHABC (fluorescent hyaluronic acid binding complex) in various cell types ${ }^{52}$ (Fig. 2c). Lectins (Box 1) such as wheat germ agglutinin (WGA) have also been used to label the surface of migrating cells and detect the formation of migrasomes on retraction fibers ${ }^{90}$. Alternative approaches have exploited migrasome-enriched transmembrane proteins such as TSPAN4 to track migrasome formation live in migrating cultured cells and during embryonic development in $D$. rerio $^{2,3,91}$. Fluorescently tagged cytosolic proteins enriched in PM-derived EVs, such as midbody remnants, can also be harnessed to track biogenesis and uptake $^{20,92}$. Immune cell synaptic microvesicle release can be studied on planar-supported lipid bilayers containing fluorescently labelled triggers of cargo loading into EVs via CLEM and STORM techniques ${ }^{53,54}$. These approaches may allow study of the molecular machinery of EV generation in an ideal setting for super-resolution microscopy.

To visualize exosome release, one successful approach is to image MVB-PM fusion. The acidic late-endosomal $\mathrm{pH}$ underlies the reason that PM fusion results in a burst of fluorescence from (super ecliptic) CD63-pHluorin ${ }^{93}$, which can be observed by live microscopy approaches, including total internal reflection fluorescence (TIRF) and spinning-disk microscopy ${ }^{47,49,50}$. This approach depends on fast acquisition times or dynamic CLEM to distinguish full MVB-PM fusion from rapid kiss-and-run motions that are inefficient in exosome release ${ }^{49}$ (Fig. 2d). CD63-pHluorin provides single-cell spatial information of release with high temporal resolution ${ }^{47,49,50}$. But this approach is best suited for flat surfaces (for example, the basolateral side of cells) and shorter time acquisitions at single-cell level, and hence less suitable than luciferase-coupled CD63 for medium- and high-throughput screens of EV biogenesis modulators ${ }^{94}$. Dual-color microscopy of dual-tagged reporters (pHluorin-CD63-mScarlet) allows MVBs to be tracked before fusion ${ }^{48}$, while other reporter combinations can unravel the molecular identity of MVBs that fuse with the $\mathrm{PM}^{50}$. However, using CD63-pHluorin to visualize MVB$\mathrm{PM}$ fusion remains challenging in vivo due to the lack of high-speed and high-resolution modalities with limited phototoxicity ${ }^{17}$.

Imaging ILV formation in MVBs to study putative exosome biogenesis processes is equally challenging, as most live approaches lack single-vesicle resolution. The induction of enlarged endosomes by overexpressing GTPase-defective Rab5 improves resolution, but alters MVB maturation and function ${ }^{95}$. Moreover, MVBs may be destined for lysosomal degradation rather than EV secretion, limiting their relevance for exosome biogenesis. The giant secretory MVB-like compartments from D. melanogaster accessory glands allow unperturbed confocal and super-resolution visualization of intracellular sorting events and colocalization analysis of fluorescently labelled cargo proteins on ILVs in vivo ${ }^{46}$, but these processes may be specific to specialized cells.

Future developments are needed to combine measurements of ILV generation, exosome release, and PM budding simultaneously; for example, using high-speed $3 \mathrm{D}$ imaging. A clever approach to visualize protein trafficking has already revealed differences in endosome- and PM-derived EV proteomes ${ }^{96}$. Understanding these processes in further detail will let us interfere with formation and/or release of EV subclasses and provide an invaluable asset in our quest to attribute specific functions to EV subtypes in vivo.

Imaging EV distribution. After EV release in vivo, the microenvironment plays a major role in EV distribution and function. Apart from EV-intrinsic factors (for example, adhesion molecules), the local 3D architecture, extracellular matrix (ECM ${ }^{97}$ and biological barriers between organs affect $\mathrm{EV}$ diffusion and influence the physiological role of EVs (Fig. 3a). As these constraints determine local retention ${ }^{47,98}$ versus distant transport and may not be fully recapitulated in vitro, the need for realistic in vivo models of $\mathrm{EV}$ distribution is clear (Fig. 3).

Although murine studies are limited mostly to organ scale ${ }^{26}$ and disclose only the 'final destination' of EVs, smaller, transparent organisms allow subcellular resolution ${ }^{19}$ and live tracking of EV diffusion and transport (Table 4). Bioluminescent labeling, radiolabeling, and metabolic labeling are compatible with the former strategy, whereas the latter typically employs fluorescent protein- and lipid-labeling strategies.

Compared with studying endogenous EVs, isolation and injection of exogenous EVs permits fine control of engineering and dosing for optimal half-life and functional ${ }^{43}$ studies. Such studies have suggested rapid removal by tissue and cell types with sustained phagocytic capacity, even within 5 min after injection ${ }^{99}$. Although EV injection does not recapitulate the earliest aspects of the EV lifespan, two recent in vivo studies demonstrated that pre-labeled injected tumor EVs did not deviate considerably in fate from physiological EVs that are endogenously released in the blood flow ${ }^{17,19}$ (Fig. 3c,d).

Yet, it is not clear whether these examples are sufficient to warrant a generalized verdict concerning all EVs and all aspects of EV biology, especially regarding mRNA transfer ${ }^{100}$. Indeed, exogenous administration incompletely mimics physiological EV release levels (unless approximated by sustained delivery methods ${ }^{101}$ ), and physiological and pathological factors that might influence endogenous EV subset(s) might be absent in vitro ${ }^{84-89}$. EV subtypes isolated from in vitro cultures, some of which would normally act locally, would also artificially reach non-physiological sites upon injection in vivo. For example, EVs involved in ECM deposition and modulation ${ }^{47,102}$ might normally act near the cell of origin, as would EVs released at immunological or neurological synapses ${ }^{35,53,103,104}$. In addition, anatomical differences in vascular permeability (for example, liver versus brain), pathological conditions affecting endothelial barrier function, or antiviral mechanisms restricting EV diffusion could alter the efficiency of EV propagation and uptake ${ }^{99,105}$. Imaging the release and biodistribution of endogenous EV subsets in vivo under various conditions will reveal how EVs cross biological barriers under physiological conditions, for which only indirect proof is currently available; for example, intravenously injected EVs in the brain $^{106,107}$. Ultimately, comparative studies of both endogenous and exogenous EV administration are needed. Studying endogenous EVs will show physiological concentrations and dynamics of EV release and biodistribution that highlight the best sites and frequencies of injection. This will help us to interpret exogenous EV studies and will permit finer control of certain EV-intrinsic variables. Together, these comparisons will inform EV targeting approaches for therapeutics.

\section{Imaging interaction and uptake of EVs by recipient cells and related functions}

The EV lifespan is often depicted as cell A releasing EVs that reach cell $\mathrm{B}$, where endocytosis and (intraluminal) cargo delivery trigger a phenotypic response. Although this communication paradigm is exciting and supported by literature, EVs can also act in an autocrine fashion or have other 'delivery-independent' extracellular functions such as ECM modulation, PM receptor engagement or transfer of EV-resident proteins to recipient cells ${ }^{108-110}$ (Fig. 4a). 
a



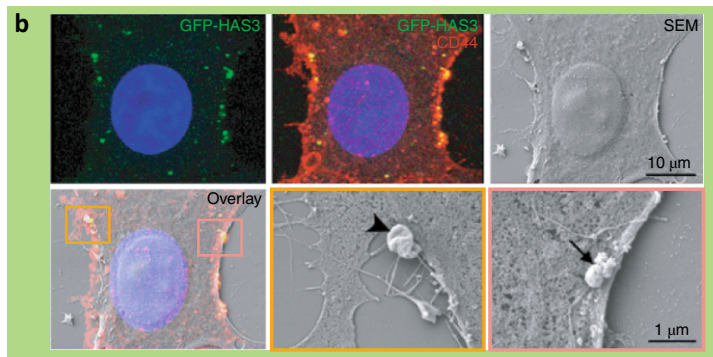
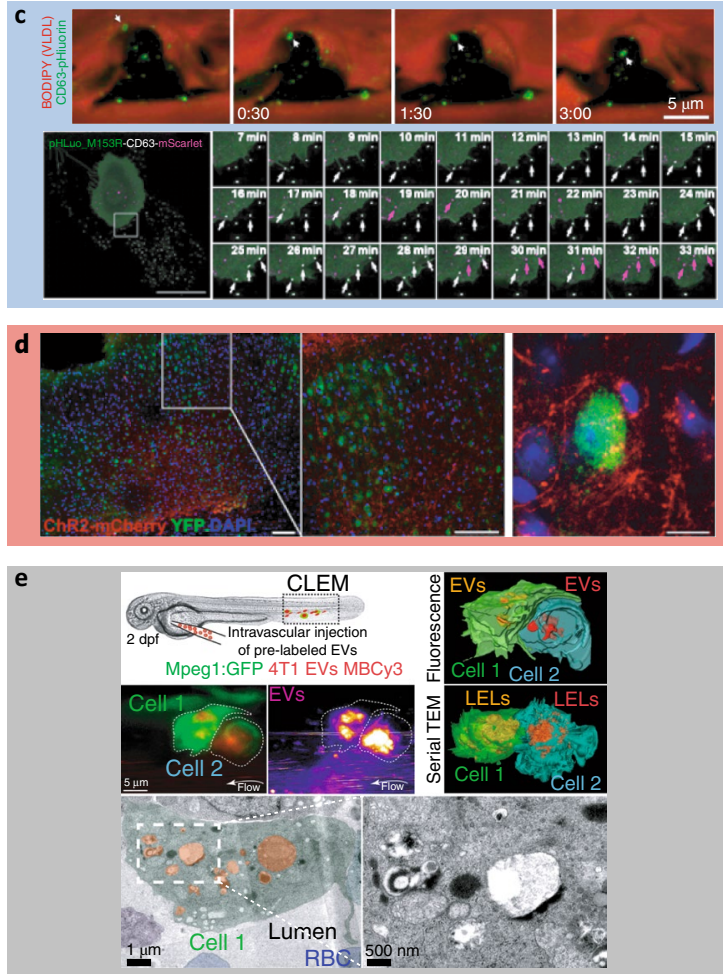

Fig. 4 | Tagging strategies to image EV interaction, uptake, and fate. a, Different tagging strategies (blue box) reveal distinct aspects of EV-cell interactions. TEV, tobacco etch virus. b-e, Imaging strategies to track the fate and functions of EVs. $\mathbf{b}$, Correlative light and scanning electron microscopy shows GFP-HAS3-labeled EVs interacting with the PM of receiving cells ${ }^{115}$. Arrowhead, large EV; arrow, cluster and individual EVs of different sizes. c, Tracking uptake of endogenous CD63-pHluorin-labeled EVs in the D. rerio vasculature ${ }^{17}$ (top); and tracking double-labeled pHluorin-CD63-mScarlet EVs inside and outside HT1080 cells ${ }^{48}$ (bottom). VLDL, very low density lipoprotein. Arrows, internalized EV (top). White arrows, external EVs; purple arrows, internal EVs (bottom). d, Ex vivo mapping of EV mRNA using a Cre recombinase strategy in the mouse brain ${ }^{18}$. e, Correlative light and electron microscopy shows Membright Cy3 lipid dye-labeled EVs accumulating in endolysosomes in D. rerio patrolling macrophages. ${ }^{19}$ dpf, days post-fertilization; LEL, late endo-lysosome; RBC, red blood cell.

Imaging interaction of EVs with recipient cells. EVs can function by engaging PM-localized receptors at the target cell membrane, such as in antigen presentation, as super-cytokines, or as carriers of morphogens and ligands for pattern recognition receptors ${ }^{42,54,63,108,111-113}$. Whereas uptake of EVs has been amply demonstrated by (live) imaging, visualization of $\mathrm{EV}$ interaction with the $\mathrm{PM}$ has been reported on just a few occasions ${ }^{60}$, and only recently with live imaging in vitro ${ }^{59,114}$ and in vivo ${ }^{17,19}$. Direct observation ${ }^{115}$ might currently be limited due to a lack of suitable reporters. Indeed, whereas most studies adding labeled EVs to target cells show intracellular accumulation rather than PM labeling, this does not preclude previous EVPM interaction, especially as functional cargo delivery appears to be a rare event from the 'bulk EV flow' perspective. For certain EVs, uptake might indeed be a prerequisite to function, but for other EVs, uptake followed by degradation could instead reflect an end-of-life event after signaling through PM receptors. To date, most reporter systems for EV function have focused on cytoplasmic cargo delivery rather than signal induction. Understanding fusion-independent EV functions thus requires combined microscopy approaches, such as CLEM (Fig. 4b), in vitro ${ }^{35,60,113}$ and in vivo ${ }^{17,19,61}$, to cover the full range from whole organism to subcellular at sufficient resolution with light-microscopy or EM ultrastructural resolution.

Imaging cellular uptake of EVs. EVs are widely reported to deliver contents into the cytoplasm of recipient cells such as signaling proteins, RNA binding proteins, genetic material, metabolites, and enzymes. However, we know little about the fusion events or transporter systems necessary for such delivery. Often, studies follow uptake in bulk, and lack the resolution to study the fate of single EVs. Recently, EM has been used to examine EV uptake in vivo ${ }^{17,19}$. Live imaging approaches can reveal other details of EV fate, such as acidification of EV-containing compartments after uptake in vitro ${ }^{48}$ and in vivo ${ }^{17}$, distinguishing 'storage' from degradation (Fig. 4c). V-ATPase (Box 1) inhibitors might be required if uptake 
and degradation are highly efficient in target cells or to facilitate detection of rare events. Note that the choice of dye (for example, lipid or genetic protein labeling) determines what is being followed after EV uptake. Over time, labels might no longer represent intact EVs, but rather the trafficking of the label itself or of lipid or protein fragments.

Imaging EV function in recipient cells. EVs elicit phenotypic responses in proximally and distally located cells. Reporter systems have been developed to visualize transfer of mRNAs ${ }^{21,22,100}$, miR$\mathrm{NAs}^{39,116}$, shRNAs ${ }^{23}$, and proteins ${ }^{117}$. Cytoplasmic delivery presupposes endosomal escape by EV-endosome fusion to avoid lysosomal degradation of EV cargos. So far, detection of cargo transfer by live imaging is limited to induction of a global signal at the cellular scale (Fig. 4d). Further resolution is needed to locate and elucidate endosomal escape, demanding new technological developments for single-molecule cargo tracking and to observe potential fusion (Box 1) of endocytosed EVs with the host membrane. Interestingly, in vivo mouse studies indicate that cargo transfer occurs at low 'efficiency' in the absence of a specific stimulus ${ }^{21,100}$. However, in certain pathological models, the functional uptake of EVs can be higher ${ }^{118}$, highlighting the need to study pathological situations in model organisms.

Several reports indicate a trophic support function of EVs via lysosomal degradation ${ }^{17,119}$. Lysosomal targeting can be studied by $\mathrm{EM}^{17,19}$ (Fig. 4e) or by live imaging using EV reporters with different acid sensitivity ${ }^{20,120}$. Live imaging in vivo revealed rapid internalization and degradation of injected or endogenous EVs by professional phagocytes (for example, macrophages and monocytes) and especially pinocytes (for example, scavenger endothelial cells). Therefore, some EVs might function without message delivery ${ }^{17,19}$. Although trophic function is not strictly incompatible with 'message transfer', a yet-unresolved question is whether EV-mediated communication is stochastic or deterministic from a donor cell perspective. Do cells release a large amount of EVs agnostically, letting the recipient cell determine whether to respond via an 'activation status' that determines cytoplasmic cargo delivery ${ }^{118}$ ? Or do cells release a limited number of 'magic bullet' EVs that are tailored for specific communication? The latter is currently supported in the immunological synapse setting ${ }^{35,53,60}$, but is perhaps less evident beyond this close cell-cell contact setting. These 'magic bullets' might be present within the main flow but possess molecular traits that promote capture, facilitate back-fusion (Box 1), or prevent degradation. Thus, tracking bulk EV flow may divert our attention from the rare EV-target cell interactions, the 'magic bullets' that do not follow bulk flow fate.

Technological strategies are important to monitor events in the transfer process ${ }^{121}$, but perhaps the most pressing need is to develop more fundamental knowledge of rare, 'magic-bullet' events. When we know the players, we can image the co-packaging of cognate molecules and targeting molecules into ILVs and EVs to follow EV lifespan events in real time, from biogenesis to target cell interactions.

\section{Conclusion}

Imaging technology has matured such that we can study most details of the EV lifespan in vivo using diverse tags and microscopy approaches, especially in optically transparent organisms. What is at stake is profound. Imaging biogenesis will distinguish EV subpopulations perhaps associated with distinct functions, and enable a firm nomenclature. By following the biodistribution of EVs in vivo, we will not only assess their capacity to cross biological barriers but also gain insight into their range of actions and their efficiency in reaching target cells previously identified in vitro. In vitro technologies can then be used to dissect mechanisms in more detail, lifting the veil around the important events that in vivo imaging has started to reveal ${ }^{73,106,122}$.
How EVs act as mediators of intercellular signaling is poorly understood. By following the fate of EVs in vivo, we will gain insight into their in vivo targets and functions. Direct imaging of the release of EV contents into recipient cells is needed to identify whether cargo transfer or signaling interaction (or both), is responsible for the effects of EVs. Although most studies focus on EV functions requiring EV uptake and cargo transfer into recipient cells, mounting evidence points towards extracellular roles for EVs involving neither uptake nor cargo delivery ${ }^{42,63,108,111,112,123}$. It is unclear how common extracellular roles are in vivo compared to intracellular functions, and whether EVs mainly act systemically rather than locally. The rapid clearance of the majority of injected EVs by the liver and spleen might indicate that many EVs function in waste disposal or trophic support. Therefore, it is important to determine the route taken by endogenous EVs in vivo and the amount of EVs necessary to impact target tissues. Following specific subclasses of EVs in vivo will aid in addressing these key questions, and reveal whether EV communication is stochastic and inefficient or rather relies on specialized EVs to transfer messages.

Knowing the in vivo characteristics of EVs, such as their half-life, biodistribution and targeting mechanisms, also supports their clinical application as biomarkers, drug carriers, or intrinsic modulators of pathological and physiological processes ${ }^{124-126}$. In vivo imaging approaches reveal the time and location of EV-subtype release and the biological fluids in which they are distributed or accumulate. This 'hot spot' mapping could optimize strategies to timely harvest the most relevant EVs for diagnosis or disease monitoring. High-resolution imaging of injected EVs purposed for drug delivery can likewise reveal EV pharmacokinetics (half-life, biodistribution, clearance), fate, and effects on recipient cells in real-time. This supports the development of engineering and administration protocols for efficient biodistribution and targeting, minimal clearance, and improved drug delivery efficiency in clinical practice. Monitoring EV dynamics in vivo will also identify drug targets for modulating EV release, uptake and degradation, influencing pharmacokinetics and EV-intrinsic functions. Thus, in vivo imaging approaches will not only provide crucial insight into fundamental aspects of the EV lifespan but will also benefit clinical development of EV-based drug delivery systems ${ }^{127}$.

The future of the field critically depends on a systematic approach comparing the pros and cons of each EV labeling and imaging strategy, in vitro and in vivo, to establish their relevance and determine good practices. We foresee development of important synergies between imaging methods and other techniques such as synthetic biology tools to investigate EV biology in vivo; for instance, by controlling and validating EV secretion and fate in vivo and to facilitate downstream analysis of specific EV subpopulations ex vivo. Imaging is now part of the toolbox of scientists studying EVs, who will work with other nanoscientists to further elucidate the biology and therapeutic applications of EVs.

Received: 12 November 2020; Accepted: 20 May 2021; Published online: 26 August 2021

\section{References}

1. Van Niel, G., D’Angelo, G. \& Raposo, G. Shedding light on the cell biology of extracellular vesicles. Nat. Rev. Mol. Cell Biol. 19, 213-228 (2018).

2. Jiang, D. et al. Migrasomes provide regional cues for organ morphogenesis during zebrafish gastrulation. Nat. Cell Biol. 21, 966-977 (2019).

3. Huang, Y. et al. Migrasome formation is mediated by assembly of micron-scale tetraspanin macrodomains. Nat. Cell Biol. 21, 991-1002 (2019).

4. Nicolás-Ávila, J. A. et al. A network of macrophages supports mitochondrial homeostasis in the heart. Cell 183, 94-109.e23 (2020).

5. Melentijevic, I. et al. C. elegans neurons jettison protein aggregates and mitochondria under neurotoxic stress. Nature 542, 367-371 (2017). 
6. Zhang, H. et al. Identification of distinct nanoparticles and subsets of extracellular vesicles by asymmetric flow field-flow fractionation. Nat. Cell Biol. 20, 332-343 (2018).

7. Bálint et al. Supramolecular attack particles are autonomous killing entities released from cytotoxic T cells. Science 368, 897-901 (2020).

8. Marki, A. et al. Elongated neutrophil-derived structures are blood-borne microparticles formed by rolling neutrophils during sepsis. J. Exp. Med. 218, e20200551 (2021).

9. Schubert, D. A brief history of adherons: the discovery of brain exosomes. Int. J. Mol. Sci. 21, 1-9 (2020).

10. Harding, C., Heuser, J. \& Stahl, P. Receptor-mediated endocytosis of transferrin and recycling of the transferrin receptor in rat reticulocytes. J. Cell Biol. 97, 329-339 (1983).

11. Raposo, G. et al. B lymphocytes secrete antigen-presenting vesicles. J. Exp. Med. 183, 1161-1172 (1996).

12. Yáñez-Mó, M. et al. Biological properties of extracellular vesicles and their physiological functions. J. Extracell. Vesicles 4, 27066 (2015)

13. Budnik, V., Ruiz-Cañada, C. \& Wendler, F. Extracellular vesicles round off communication in the nervous system. Nat. Rev. Neurosci. 17, 160-172 (2016).

14. Stahl, P. D. \& Raposo, G. Extracellular vesicles: exosomes and microvesicles, integrators of homeostasis. Physiology 34, 169-177 (2019).

15. Boulanger, C. M., Loyer, X., Rautou, P.-E. \& Amabile, N. Extracellular vesicles in coronary artery disease. Nat. Rev. Cardiol. 14, 259-272 (2017).

16. Jeppesen, D. K. et al. Reassessment of exosome composition. Cell 177, 428-445.e18 (2019).

17. Verweij, F. J. et al. Live tracking of inter-organ communication by endogenous exosomes in vivo. Dev. Cell 48, 573-589.e4 (2019).

18. van der Vos, K. E. et al. Directly visualized glioblastoma-derived extracellular vesicles transfer RNA to microglia/macrophages in the brain. Neuro. Oncol. 18, 58-69 (2016).

19. Hyenne, V. et al. Studying the fate of tumor extracellular vesicles at high spatiotemporal resolution using the zebrafish embryo. Dev. Cell $\mathbf{4 8}$, 554-572.e7 (2019).

20. Fazeli, G., Trinkwalder, M., Irmisch, L. \& Wehman, A. M. C. elegans midbodies are released, phagocytosed and undergo LC3-dependent degradation independent of macroautophagy. J. Cell Sci. 129, 3721-3731 (2016).

21. Ridder, K. et al. Extracellular vesicle-mediated transfer of genetic information between the hematopoietic system and the brain in response to inflammation. PLoS Biol. 12, e1001874 (2014).

22. Zomer, A. et al. In vivo imaging reveals extracellular vesicle-mediated phenocopying of metastatic behavior. Cell 161, 1046-1057 (2015).

23. de Jong, O. G. et al. A CRISPR-Cas9-based reporter system for single-cell detection of extracellular vesicle-mediated functional transfer of RNA. Nat. Commun. 11, 1113 (2020).

24. Gonçalves, M. S. T. Fluorescent labeling of biomolecules with organic probes. Chem. Rev. 109, 190-212 (2009).

25. Pužar Dominkuš, P. et al. PKH26 labeling of extracellular vesicles: characterization and cellular internalization of contaminating PKH26 nanoparticles. Biochim. Biophys. Acta Biomembr. 1860, 1350-1361 (2018).

26. Corso, G. et al. Systematic characterization of extracellular vesicles sorting domains and quantification at the single molecule-single vesicle level by fluorescence correlation spectroscopy and single particle imaging. J. Extracell. Vesicles 8, 1663043 (2019).

27. Collot, M. et al. MemBright: a family of fluorescent membrane probes for advanced cellular imaging and neuroscience. Cell Chem. Biol. 26, 600-614.e7 (2019)

28. Dehghani, M., Gulvin, S. M., Flax, J. \& Gaborski, T. R. Exosome labeling by lipophilic dye PKH26 results in significant increase in vesicle size. Preprint at bioRxiv https://doi.org/10.1101/532028 (2019).

29. Kuffler, D. P. Long-term survival and sprouting in culture by motoneurons isolated from the spinal cord of adult frogs. J. Comp. Neurol. 302, 729-738 (1990).

30. Gray, W. D., Mitchell, A. J. \& Searies, C. D. An accurate, precise method for general labeling of extracellular vesicles. MethodsX 2, 488-496 (2015).

31. Chuo, S. T.-Y., Chien, J. C.-Y. \& Lai, C. P.-K. Imaging extracellular vesicles: current and emerging methods. J. Biomed. Sci. 25, 91 (2018).

32. Mckinnon, K. M. et al. Labeling extracellular vesicles for nanoscale flow cytometry. Sci. Rep. 7, 1878 (2017).

33. Liao, Z. et al. Acetylcholinesterase is not a generic marker of extracellular vesicles. J. Extracell. Vesicles 8, 1628592 (2019).

34. Lai, C. P. et al. Visualization and tracking of tumour extracellular vesicle delivery and RNA translation using multiplexed reporters. Nat. Commun. 6 7029 (2015).

35. Mittelbrunn, M. et al. Unidirectional transfer of microRNA-loaded exosomes from T cells to antigen-presenting cells. Nat. Commun. 2, 282 (2011).

36. Mathieu, M., Martin-Jaular, L., Lavieu, G. \& Théry, C. Specificities of secretion and uptake of exosomes and other extracellular vesicles for cell-to-cell communication. Nat. Cell Biol. 21, 9-17 (2019).
37. Badr, C. E. \& Tannous, B. A. Bioluminescence imaging: progress and applications. Trends Biotechnol. 29, 624-633 (2011).

38. Takahashi, Y. et al. Visualization and in vivo tracking of the exosomes of murine melanoma B16-BL6 cells in mice after intravenous injection. J. Biotechnol. 165, 77-84 (2013).

39. Lai, C. P. et al. Dynamic biodistribution of extracellular vesicles in vivo using a multimodal imaging reporter. ACS Nano. 8, 483 (2014).

40. $\mathrm{Wu}, \mathrm{A}$. Y. T. et al. Multiresolution imaging using bioluminescence resonance energy transfer identifies distinct biodistribution profiles of extracellular vesicles and exomeres with redirected tropism. Adv. Sci. 7, 2001467 (2020).

41. Hoshino, A. et al. Tumour exosome integrins determine organotropic metastasis. Nature 527, 329-335 (2015).

42. Chen, G. et al. Exosomal PD-L1 contributes to immunosuppression and is associated with anti-PD-1 response. Nature 560, 382-386 (2018).

43. Ghoroghi, S. et al. Ral GTPases promote breast cancer metastasis by controlling biogenesis and organ targeting of exosomes. eLife 10, 61539 (2021).

44. Zaborowski, M. P. et al. Membrane-bound Gaussia luciferase as a tool to track shedding of membrane proteins from the surface of extracellular vesicles. Sci. Rep. 9, 17387 (2019).

45. Shinoda, H., Shannon, M. \& Nagai, T. Fluorescent proteins for investigating biological events in acidic environments. Int. J. Mol. Sci. 19, 1548 (2018).

46. Fan, S. et al. Glutamine deprivation alters the origin and function of cancer cell exosomes. EMBO J. 39, e103009 (2020).

47. Sung, B. H., Ketova, T., Hoshino, D., Zijlstra, A. \& Weaver, A. M. Directional cell movement through tissues is controlled by exosome secretion. Nat. Commun. 6, 7164 (2015).

48. Sung, B. H. et al. A live cell reporter of exosome secretion and uptake reveals pathfinding behavior of migrating cells. Nat. Commun. 11, 2092 (2020).

49. Verweij, F. J. et al. Quantifying exosome secretion from single cells reveals a modulatory role for GPCR signaling. J. Cell Biol. 217, 1129-1142 (2018).

50. Bebelman, M. P. et al. Real-time imaging of multivesicular body-plasma membrane fusion to quantify exosome release from single cells. Nat. Protoc. 15, 102-121 (2020).

51. Beer, K. B. et al. Degron-tagged reporters probe membrane topology and enable the specific labelling of membrane-wrapped structures. Nat. Commun. 10, 3490 (2019)

52. Mustonen, A. M. et al. First in vivo detection and characterization of hyaluronan-coated extracellular vesicles in human synovial fluid. J. Orthop. Res. 34, 1960-1968 (2016).

53. Choudhuri, K. et al. Polarized release of T-cell-receptor-enriched microvesicles at the immunological synapse. Nature 507, 118-123 (2014).

54. Saliba, D. G. et al. Composition and structure of synaptic ectosomes exporting antigen receptor linked to functional CD40 ligand from helper T cells. eLife 8, e47528 (2019).

55. Ambrose, A. R., Hazime, K. S., Worboys, J. D., Niembro-Vivanco, O. \& Davis, D. M. Synaptic secretion from human natural killer cells is diverse and includes supramolecular attack particles. Proc. Natl Acad. Sci. USA. 117, 23717-23720 (2020).

56. Kanwar, S. S., Dunlay, C. J., Simeone, D. M. \& Nagrath, S. Microfluidic device (ExoChip) for on-chip isolation, quantification and characterization of circulating exosomes. Lab Chip 14, 1891-1900 (2014).

57. Icha, J., Weber, M., Waters, J. C. \& Norden, C. Phototoxicity in live fluorescence microscopy, and how to avoid it. BioEssays 39, 1700003 (2017)

58. Spikes, J. D. Photosensitization in mammalian cells. in Photoimmunology (eds Parrish, J. A. et al.) 23-49 (Springer, 1983).

59. Elgamal, S., Colombo, F., Cottini, F., Byrd, J. C. \& Cocucci, E. Imaging intercellular interaction and extracellular vesicle exchange in a co-culture model of chronic lymphocytic leukemia and stromal cells by lattice light-sheet fluorescence microscopy. Methods Enzymol. 645, 79-107 (2020).

60. Buschow, S. I. et al. MHC II in dendritic cells is targeted to lysosomes or T cell-induced exosomes via distinct multivesicular body pathways. Traffic 10, 1528-1542 (2009).

61. Hurbain, I. et al. Microvilli-derived extracellular vesicles govern morphogenesis in Drosophila wing epithelium. Preprint at bioRxiv https://doi.org/10.1101/2020.11.01.363697 (2020).

62. González-Méndez, L. et al. Polarized sorting of Patched enables cytonememediated Hedgehog reception in the Drosophila wing disc. EMBO J. 39 , e103629 (2020)

63. Gross, J. C., Chaudhary, V., Bartscherer, K. \& Boutros, M. Active Wnt proteins are secreted on exosomes. Nat. Cell Biol. 14, 1036-1045 (2012)

64. Matusek, T. et al. The ESCRT machinery regulates the secretion and long-range activity of Hedgehog. Nature 516, 99-103 (2014).

65. Gradilla, A. C. et al. Exosomes as Hedgehog carriers in cytoneme-mediated transport and secretion. Nat. Commun. 5, 5649 (2014). 
66. Tassetto, M., Kunitomi, M. \& Andino, R. Circulating immune cells mediate a systemic rnai-based adaptive antiviral response in drosophila. Cell 169, 314-325.e13 (2017).

67. Wang, J. et al. C. elegans ciliated sensory neurons release extracellular vesicles that function in animal communication. Curr. Biol. 24, 519-525 (2014).

68. Wehman, A. M., Poggioli, C., Schweinsberg, P., Grant, B. D. \& Nance, J. The P4-ATPase TAT-5 inhibits the budding of extracellular vesicles in C. elegans embryos. Curr. Biol. 21, 1951-1959 (2011).

69. Hyenne, V. et al. hRAL-1 controls multivesicular body biogenesis and exosome secretion. J. Cell Biol. 211, 27-37 (2015).

70. Härkönen, K. et al. CD44s Assembles hyaluronan coat on filopodia and extracellular vesicles and induces tumorigenicity of MKN74 gastric carcinoma. Cells Cells 8, 276 (2019).

71. Verweij, F. J., Hyenne, V., Van Niel, G. \& Goetz, J. G. Extracellular vesicles: catching the light in zebrafish. Trends Cell Biol. 29, 770-776 (2019).

72. Abels, E. R. et al. Glioblastoma-associated microglia reprogramming is mediated by functional transfer of extracellular miR-21. Cell Rep. 28, 3105-3119.e7 (2019).

73. Gupta, D. et al. Quantification of extracellular vesicles in vitro and in vivo using sensitive bioluminescence imaging. J. Extracell. vesicles 9, 1800222 (2020).

74. Men, Y. et al. Exosome reporter mice reveal the involvement of exosomes in mediating neuron to astroglia communication in the CNS. Nat. Commun. 10, 4136 (2019).

75. Baumeister, R. \& Ge, L. The worm in us - Caenorhabditis elegans as a model of human disease. Trends Biotechnol. 20, 147-148 (2002).

76. Howe, $\mathrm{K}$. et al. The zebrafish reference genome sequence and its relationship to the human genome. Nature 496, 498-503 (2013).

77. Fortini, M. E., Skupski, M. P., Boguski, M. S. \& Hariharan, I. K. A survey of human disease gene counterparts in the Drosophila genome. J. Cell Biol. 150, F23-F30 (2000)

78. Santoriello, C. \& Zon, L. I. Hooked! Modeling human disease in zebrafish. J. Clin. Invest. 122, 2337-2343 (2012).

79. Caygill, E. E. \& Brand, A. H. The GAL4 system: a versatile system for the manipulation and analysis of gene expression. in Drosophila: Methods and Protocols 2nd edn (ed. Dahmann, C.) 33-52 (Humana Press, 2016).

80. Port, F. et al. A large-scale resource for tissue-specific CRISPR mutagenesis in Drosophila. eLife 9, e53865 (2020).

81. Albadri, S., De Santis, F., Di Donato, V. \& Del Bene, F. CRISPR/ Cas9-mediated knockin and knockout in zebrafish. in Genome Editing in Neurosciences. Research and Perspectives in Neurosciences (eds. Jaenisch, R., Zhang, F. \& Gage, F.) 41-49 (2017)

82. Muntasell, A., Berger, A. C. \& Roche, P. A. T cell-induced secretion of MHC class II-peptide complexes on B cell exosomes. EMBO J. 26, 4263-4272 (2007).

83. Lachenal, G. et al. Release of exosomes from differentiated neurons and its regulation by synaptic glutamatergic activity. Mol. Cell. Neurosci. 46, 409-418 (2011).

84. Li, J. et al. Serum-free culture alters the quantity and protein composition of neuroblastoma-derived extracellular vesicles. J. Extracell. vesicles 4 , 26883 (2015)

85. Rocha, S. et al. 3D cellular architecture affects microRNA and protein cargo of extracellular vesicles. Adv. Sci. 6, 1800948 (2019).

86. Thippabhotla, S., Zhong, C. \& He, M. 3D cell culture stimulates the secretion of in vivo like extracellular vesicles. Sci. Rep. 9, 13012 (2019).

87. Cao, J. et al. Three-dimensional culture of MSCs produces exosomes with improved yield and enhanced therapeutic efficacy for cisplatin-induced acute kidney injury. Stem Cell Res. Ther. 11, 206 (2020).

88. Kim, M., Yun, H.-W., Park, D. Y., Choi, B. H. \& Min, B.-H. Three-dimensional spheroid culture increases exosome secretion from mesenchymal stem cells. Tissue Eng. Regen. Med. 15, 427-436 (2018).

89. Lehrich, B. M., Liang, Y. \& Fiandaca, M. S. Foetal bovine serum influence on in vitro extracellular vesicle analyses. J. Extracell. Vesicles 10, e12061 (2021).

90. Chen, L., Ma, L. \& Yu, L. WGA is a probe for migrasomes. Cell Discov. 5, 13 (2019).

91. Ma, L. et al. Discovery of the migrasome, an organelle mediating release of cytoplasmic contents during cell migration. Cell Res. 25, 24-38 (2015).

92. Addi, C. et al. The Flemmingsome reveals an ESCRT-to-membrane coupling via ALIX/syntenin/syndecan-4 required for completion of cytokinesis. Nat. Commun. 11, 1941 (2020).

93. Miesenbock, G., De Angelis, D. A. \& Rothman, J. E. Visualizing secretion and synaptic transmission with $\mathrm{pH}$-sensitive green fluorescent proteins. Nature 394, 192-195 (1998).

94. Cashikar, A. G. \& Hanson, P. I. A cell-based assay for CD63-containing extracellular vesicles. PLoS One 14, e0220007 (2019).

95. Wegner, C. S. et al. Ultrastructural characterization of giant endosomes induced by GTPase-deficient Rab5. Histochem. Cell Biol. 133, 41-55 (2010).
96. Mathieu, M. et al. Specificities of exosome versus small ectosome secretion revealed by live intracellular tracking of CD63 and CD9. Nat. Commun. https://doi.org/10.1038/s41467-021-24384-2 (2021).

97. Lenzini, S., Bargi, R., Chung, G. \& Shin, J. W. Matrix mechanics and water permeation regulate extracellular vesicle transport. Nat. Nanotechnol. 15, 217-223 (2020).

98. Mu, W., Rana, S. \& Zöller, M. Host matrix modulation by tumor exosomes promotes motility and invasiveness. Neoplasia 15, 875-IN4 (2013).

99. Wiklander, O. P. B. et al. Extracellular vesicle in vivo biodistribution is determined by cell source, route of administration and targeting. $J$. Extracell. Vesicles 4, 26316 (2015).

100. Ridder, K. et al. Extracellular vesicle-mediated transfer of functional RNA in the tumor microenvironment. Oncoimmunology 4, e1008371 (2015).

101. Riau, A. K., Ong, H. S., Yam, G. H. F. \& Mehta, J. S. Sustained delivery system for stem cell-derived exosomes. Front. Pharmacol. 10, 1368 (2019)

102. Rilla, K. et al. Extracellular vesicles are integral and functional components of the extracellular matrix. Matrix Biol. s 75-76, 201-219 (2019).

103. Pastuzyn, E. D. et al. The neuronal gene arc encodes a repurposed retrotransposon gag protein that mediates intercellular RNA transfer. Cell 172, 275-288.e18 (2018).

104. Ashley, J. et al. Retrovirus-like Gag protein Arcl binds RNA and traffics across synaptic boutons. Cell 172, 262-274.e11 (2018).

105. Edgar, J. R., Manna, P. T., Nishimura, S., Banting, G. \& Robinson, M. S. Tetherin is an exosomal tether. eLife 5, e17180 (2016).

106. Morad, G. et al. Tumor-derived extracellular vesicles breach the intact blood-brain barrier via transcytosis. ACS Nano. 13, 13853 (2019).

107. Alvarez-Erviti, L. et al. Delivery of siRNA to the mouse brain by systemic injection of targeted exosomes. Nat. Biotechnol. 29, 341-345 (2011).

108. Denzer, K. et al. Follicular dendritic cells carry MHC class II-expressing microvesicles at their surface. J. Immunol. 165, 1259-1265 (2000).

109. Gao, L. et al. Tumor-derived exosomes antagonize innate antiviral immunity. Nat. Immunol. 19, 233-245 (2018).

110. Vilcaes, A. A., Chanaday, N. L. \& Kavalali, E. T. Interneuronal exchange and functional integration of synaptobrevin via extracellular vesicles. Neuron 109, 971-983.e5 (2021).

111. Ko, S. Y. et al. Cancer-derived small extracellular vesicles promote angiogenesis by heparin-bound, bevacizumab-insensitive VEGF, independent of vesicle uptake. Commun. Biol. 2, 386 (2019).

112. Neumann, C. J. \& Cohen, S. M. Long-range action of Wingless organizes the dorsal-ventral axis of the Drosophila wing. Development 124, 871-880 (1997)

113. Tkach, M. et al. Qualitative differences in T-cell activation by dendritic cell-derived extracellular vesicle subtypes. EMBO J. 36, 3012-3028 (2017)

114. Heusermann, W. et al. Exosomes surf on filopodia to enter cells at endocytic hot spots and shuttle within endosomes to scan the ER. J. Cell Biol. 213, 173-184 (2016).

115. Arasu, U. T., Härkönen, K., Koistinen, A. \& Rilla, K. Correlative light and electron microscopy is a powerful tool to study interactions of extracellular vesicles with recipient cells. Exp. Cell. Res. 376, 149-158 (2019).

116. Thomou, T. et al. Adipose-derived circulating miRNAs regulate gene expression in other tissues. Nature 542, 450-455 (2017).

117. Sterzenbach, U. et al. Engineered exosomes as vehicles for biologically active proteins. Mol. Ther. 25, 1269-1278 (2017).

118. Kur, I.-M. et al. Neuronal activity triggers uptake of hematopoietic extracellular vesicles in vivo. PLoS Biol. 18, e3000643 (2020).

119. Frühbeis, C. et al. Neurotransmitter-triggered transfer of exosomes mediates oligodendrocyte-neuron communication. PLoS Biol. 11, e1001604 (2013).

120. Khmelinskii, A. et al. Incomplete proteasomal degradation of green fluorescent proteins in the context of tandem fluorescent protein timers. Mol. Biol. Cell 27, 360-370 (2016).

121. Joshi, B. S., De Beer, M. A., Giepmans, B. N. G. \& Zuhorn, I. S. Endocytosis of extracellular vesicles and release of their cargo from endosomes. ACS Nano. 14, 32 (2020).

122. Cao, H. et al. In vivo real-time imaging of extracellular vesicles in liver regeneration via aggregation-induced emission luminogens. ACS Nano. 13, 3522-3533 (2019).

123. Webber, J. P. et al. Differentiation of tumour-promoting stromal myofibroblasts by cancer exosomes. Oncogene 34, 290-302 (2015).

124. Lener, T. et al. Applying extracellular vesicles based therapeutics in clinical trials - an ISEV position paper. J. Extracell. vesicles 4, 30087 (2015).

125. Fais, S. et al. Evidence-Based clinical use of nanoscale extracellular vesicles in nanomedicine. ACS Nano. 10, 3886-3899 (2016).

126. Kalluri, R. \& LeBleu, V. S. The biology, function, and biomedical applications of exosomes. Science 367, eaau6977 (2020).

127. Androuin A., Verweij F.J. \& Van Niel, G. Zebrafish as a preclinical model for extracellular vesicle-based therapeutic development. Adv. Drug Deliv. Rev. https://doi.org/10.1016/j.addr.2021.05.025 (2021).

128. Liégeois, S., Benedetto, A., Garnier, J.-M., Schwab, Y. \& Labouesse, M. The V0-ATPase mediates apical secretion of exosomes containing Hedgehog-related proteins in Caenorhabditis elegans. J. Cell Biol. 173, 949-961 (2006) 
129. Koles, K. et al. Mechanism of evenness interrupted (Evi)-exosome release at synaptic boutons. J. Biol. Chem. 287, 16820-16834 (2012).

130. Corrigan, L. et al. BMP-regulated exosomes from male reproductive glands reprogram female behavior. J. Cell Biol. 206, 671-688 (2014).

131. Wolf, $P$. The nature and significance of platelet products in human plasma. Br. J. Haematol. 13, 269-288 (1967).

132. Nunez, E. A., Wallis, J. \& Gershon, M. D. Secretory processes in follicular cells of the bat thyroid. III. The occurrence of extracellular vesicles and colloid droplets during arousal from hibernation. Am. J. Anat. 141, 179-201 (1974).

133. Trams, E. G., Lauter, C. J., Norman Salem, J. \& Heine, U. Exfoliation of membrane ecto-enzymes in the form of micro-vesicles. Biochim. Biophys. Acta - Biomembr. 645, 63-70 (1981).

134. Johnstone, R. M., Bianchini, A. \& Teng, K. Reticulocyte maturation and exosome release: transferrin receptor containing exosomes shows multiple plasma membrane functions. Blood 74, 1844-1851 (1989)

135. Heijnen, H. F., Schiel, A. E., Fijnheer, R., Geuze, H. J. \& Sixma, J. J. Activated platelets release two types of membrane vesicles: microvesicles by surface shedding and exosomes derived from exocytosis of multivesicular bodies and alpha-granules. Blood 94, 3791-3799 (1999).

136. Yang, T. et al. Exosome delivered anticancer drugs across the bloodbrain barrier for brain cancer therapy in Danio rerio. Pharm. Res. 32, 2003-2014 (2015).

\section{Acknowledgements}

The authors acknowledge financial support from the International Society for Extracellular Vesicles, the French Society of Extracellular vesicles, the Société Française des Microscopies, the ITMO BCDE for their support for the organization of the ISEV workshop 'EV imaging in vivo' that provided the basis for this review. We thank the workshop organizing committee members G.D.A., V.H., E.-M.K.-A., X.L., and K.W. for their help. We thank P. Stahl (Washington University School of Medicine, USA) for stimulating discussions and insight. F.J.V. is supported by INCa 2019-125, E.B.C. thanks M. Dustin for support through ERC AdG 670930. D.R.F.C. is supported by the BBSRC (BB/P006205/1) and Cancer Research UK (A28052). V.H. and J.G.G. are funded by La Ligue contre le Cancer, by INCa (PLBIO19-291), by Plan Cancer (Nanotumor) and through institutional funds from University of Strasbourg and INSERM. K.R. is supported by the UEF Cell and Tissue Imaging Unit, Biocenter Kuopio and Biocenter Finland. We apologize to colleagues for any relevant work that could not be cited due to space restrictions.

\section{Competing interests}

D.R.F.C. is employed by Evox Therapeutics. S.E.A. serves on the Scientific Advisory Board of EVOX Therapeutics. All other authors have no competing interests.

\section{Additional information}

Correspondence should be addressed to F.J.V. or G.v.N.

Peer review information Rita Strack was the primary editor on this article and managed its editorial process and peer review in collaboration with the rest of the editorial team.

Reprints and permissions information is available at www.nature.com/reprints.

Publisher's note Springer Nature remains neutral with regard to jurisdictional claims in published maps and institutional affiliations.

(c) Springer Nature America, Inc. 2021 